\title{
A model of the natural history of screen-detected prostate cancer, and the effect of radical treatment on overall survival
}

\author{
C Parker*,', D Muston², J Melia², S Moss² and D Dearnaley' \\ 'Academic Unit of Radiotherapy \& Oncology, Institute of Cancer Research and Royal Marsden NHS Foundation Trust, Downs Road, Sutton SM2 5PT, UK; \\ ${ }^{2}$ Cancer Screening Evaluation Unit, Institute of Cancer Research, Downs Road, Sutton SM2 5PT, UK
}

\begin{abstract}
The lead time and overdetection associated with prostate-specific antigen (PSA) screening, and generational improvements in allcause mortality, make prostate cancer outcome studies from the pre-PSA era difficult to interpret in a contemporary setting. We developed a competing-risks hazard model to estimate the natural history of screen-detected prostate cancer, and the impact of radical treatment on overall survival. The model of hazard of mortality was fitted to clinical outcome data from the pre-PSA era, and the effects of screening, generational mortality improvements and radical treatment were incorporated. Sensitivities to the choice of baseline data and values of key parameters were assessed. Lead-time estimates in men diagnosed aged 55-59 years were I4.I, 9.3 and 5.0 years for men with Gleason scores $<7,7$ and $>7$, respectively, assuming biennial screening with $100 \%$ attendance. Central estimates of 15 -year prostate cancer mortality for conservative management of screen-detected prostate cancer ranged from 0 to $2 \%$ for Gleason scores $<7,9$ to $31 \%$ for Gleason score 7 and 28-72\% for Gleason scores $>7$. For men aged $55-59$ years at diagnosis, the predicted absolute 15-year survival benefit from curative treatment was 0,12 and $26 \%$ for men with Gleason scores $<7,7$ and $>7$, respectively. Estimates of the survival benefit of radical treatment were relatively insensitive to values of key parameters. The case for curative treatment, rather than conservative management, of screen-detected localised prostate cancer is strongest in men with high-grade disease. This conclusion contrasts with current patterns of care. British Journal of Cancer (2006) 94, I36I-1368. doi:I0.1038/sj.bjc.6603105 www.bjcancer.com

Published online 25 April 2006

(C) 2006 Cancer Research UK
\end{abstract}

Keywords: prostate cancer; model; outcomes

For most invasive cancers, effective curative treatment makes all the difference between life and death. While prostate cancer can follow an aggressive and ultimately fatal course, similar to that of other cancers, a significant proportion of cases will behave in an indolent fashion, with no impact either on health or longevity, even in the absence of treatment. This effect is compounded by competing causes of mortality in the typical age distribution affected by prostate cancer. Partly for these reasons, it has been difficult to establish whether radical treatment of prostate cancer improves overall survival, and, if so, by how much.

Retrospective comparisons of curative treatment $v s$ conservative management of prostate cancer are of limited value, given the likelihood of significant confounding variables. There are only two published randomised controlled trials comparing expectant management and definitive treatment of prostate cancer. Of these, one, which included just 142 patients, is too small for meaningful analysis (Iversen et al, 1995). The other, the Scandinavian Prostatic Cancer Group Study Number 4, which opened in 1989, randomised 695 men with localised disease between radical prostatectomy and watchful waiting (Bill-Axelson et al, 2005). Patients were aged less than 75 years, with well or moderately differentiated, clinically

*Correspondence: Dr C Parker; E-mail: chris.parker@rmh.nhs.uk Received 13 January 2006; revised 23 March 2006; accepted 23 March 2006; published online 25 April 2006 localised prostate cancer, a prostate-specific antigen (PSA) of less than $50 \mathrm{ng} \mathrm{ml}^{-1}$ and a life expectancy of at least 10 years. At a median follow-up of 8.2 years, randomisation to radical prostatectomy was associated with improved disease-specific mortality, with a hazard ratio of $0.56(95 \% \mathrm{CI}: 0.36-0.88 ; P=0.01)$, which translated into an absolute overall survival benefit of 5.3\% (95\% CI: -0.3 to $11.0 \%$ ) at 10 years. Although the Scandinavian trial provides the best available data comparing conservative management and curative treatment, there are very major differences between Scandinavian practice during the early 1990s and contemporary clinical practice. In particular, the case-mix in the trial was typical of clinically detected prostate cancer from the prePSA era, with only $12 \%$ of patients having stage T1c disease. It is therefore unclear how the results should be applied to men with screen-detected prostate cancer.

Prostate-specific antigen screening results in overdetection (of cases that would not otherwise have been detected) and introduces a lead time (the time difference between screen detection and clinical detection in the absence of screening), which may be of the order of 10 years or more (Draisma et al, 2003). It follows that, in the absence of treatment, the natural history of screen-detected prostate cancer will appear more favourable than that of clinically detected prostate cancer from the pre-PSA era. This is an important consideration for men faced with the choice between conservative management and curative treatment. In comparison with clinically detected disease, men with screen-detected cancers will have longer 
to endure any adverse effects of curative treatment, and longer to wait for any beneficial effect on survival to emerge.

There are two ongoing randomised trials that aim to compare curative treatment with conservative management in PSA screendetected disease. The Prostate Cancer Intervention versus Observation Trial (PIVOT) compares radical prostatectomy $v$ s watchful waiting, and is now closed to recruitment with 731 patients entered (Wilt and Brawer, 1995). The Prostate testing for cancer and Treatment (ProtecT) study compares radical prostatectomy, external beam radiotherapy and active monitoring, and aims to recruit over 2000 patients (Donovan et al, 2003). The longterm mortality outcomes from PIVOT and the ProtecT trial will provide the best data concerning the effectiveness of curative treatment of screen-detected disease, but will not be available for some years. In the meantime, knowledge of the potential impact of treatment on survival would be of great value to men found to have screen-detected prostate cancer, and who are faced with the decision whether or not to have curative treatment. We have therefore undertaken a modelling exercise to estimate the natural history of screen-detected prostate cancer, and the impact of curative treatment on overall survival.

\section{METHODS}

We constructed a competing-risks model of hazard from mortality to describe the natural history of prostate cancer from the pre-PSA era, using terminology and results outlined in Anderson et al (2002) (step one). We then adapted this model to incorporate the lead time and the probability of overdetection associated with PSA screening (step two), and generational improvements in all-cause mortality (step three). We used this model, together with evidence from the Scandinavian Prostatic Cancer Group Study (Bill-Axelson et al, 2005), to make projections of the effect of curative treatment on overall survival in contemporary patients with screen-detected localised prostate cancer (step four). This approach is summarised in Figure 1.

\section{Step one: constructing the competing risks model}

The hazard due to other-cause mortality under the given treatment was allowed to vary with time by assuming a Weibull survival distribution, with scale parameter $\lambda_{\mathrm{O}}$ and shape parameter $\gamma$ : $h_{\mathrm{O}}(t)=\lambda_{\mathrm{O}} \gamma t^{\gamma-1}$. Disease-specific mortality was assumed, for simplicity, to provide a constant hazard and therefore an exponential survival distribution, with single parameter $\lambda_{\mathrm{P}}: h_{\mathrm{P}}(t)=\lambda_{\mathrm{P}}$, where $t$ represents time since diagnosis. The total hazard is the sum of the disease-specific and other-cause hazards: $h(t)=h_{\mathrm{P}}(t)+h_{\mathrm{O}}(t)$. The model therefore requires estimates of three model parameters.

Our model parameterisation does not directly account for age and Gleason score, which are important covariates in modelling survival from prostate cancer, so it was necessary to stratify the model by these variables. To fit the model, we therefore required long-term survival outcome data from conservative management of prostate cancer, stratified by age group and Gleason score. Average or smoothed proportions (so as to eliminate random within-group variation) of the cohort alive, dead due to prostate cancer and dead due to other causes at a given time point would be sufficient to calculate two of the three parameters in the model, conditional on a value for the other.

We reviewed the literature systematically for research that would provide the necessary cohort survival data. Only one source was found: Albertsen et al $(1998,2005)$ monitored the survival of

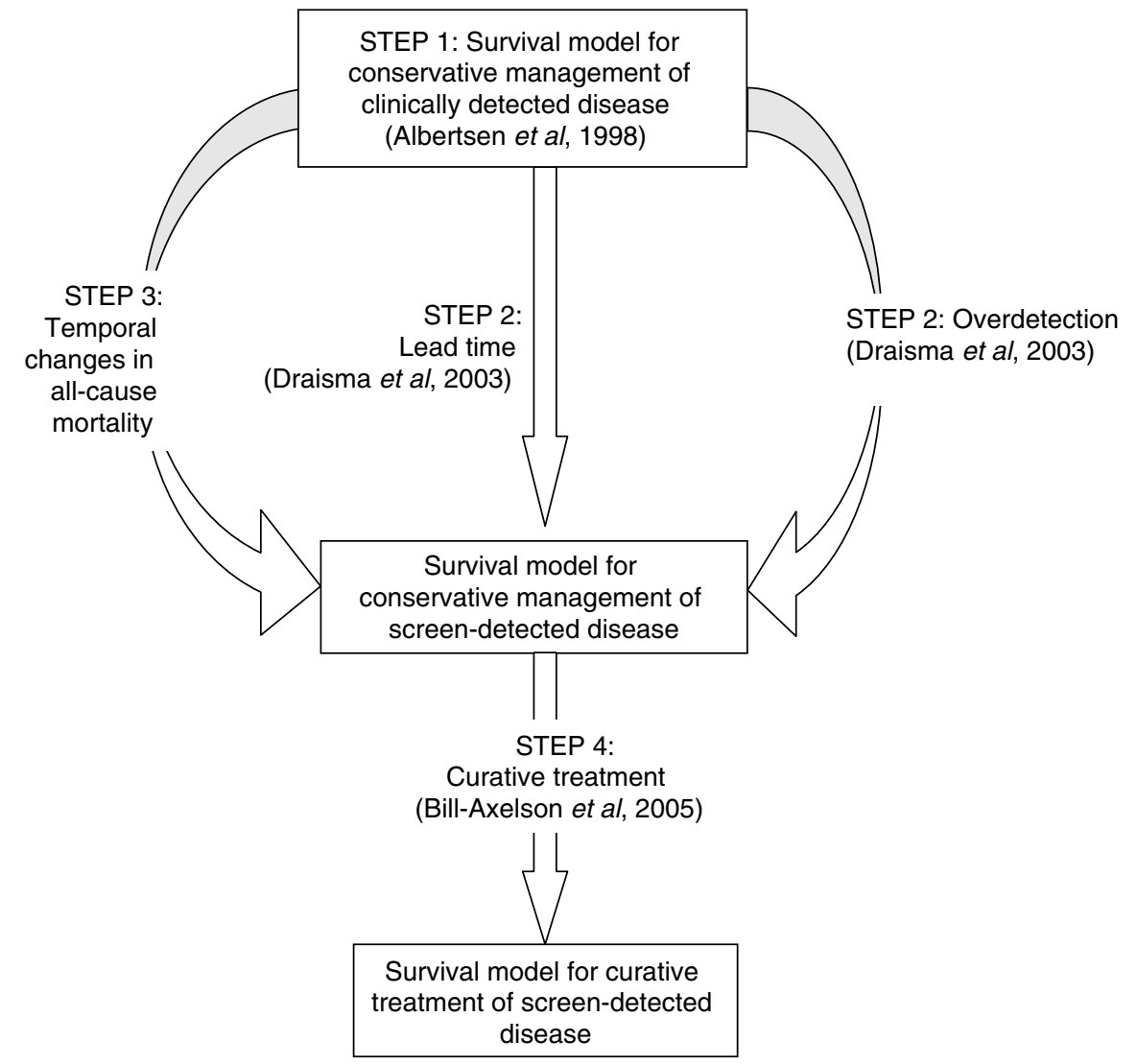

Figure I Overview of methods. 
767 men from the Connecticut Tumor Registry with localised prostate cancer diagnosed between 1971 and 1984, treated conservatively and followed up for a median of 24 years. Longterm survival probabilities fitted by proportional hazards statistical methods (Poisson regression over 15 years) were provided (Table 3 of (Albertsen et al, 1998)), stratified by age at diagnosis (within 5 years) and biopsy Gleason score $(2-4,5,6,7,8-10)$.

Parameter $\gamma$ controls the rate of increase of other-cause hazard over time rather than, directly, the level of a hazard, so is of less interest than other parameters. We therefore decided to condition our estimates for $\lambda_{\mathrm{O}}$ and $\lambda_{\mathrm{P}}$ on a value for $\gamma$. The value for $\gamma$ was estimated by fitting a single-risk Weibull model to 10000 survival periods simulated from period analyses of three US life tables $(9-11)$, with initial ages sampled uniformly between 55 and 74 years inclusive. Model A assumed constant scale and shape parameters across all age groups, model B additionally assumed interaction between the scale parameter and age group and model $\mathrm{C}$ additionally assumed interaction between the shape parameter and age group. Fitted parameter values are shown in Table 1.

Being period life tables, it is uncertain how fitted parameter values might relate to survival in a cohort. However, a single value of 1.8 was chosen for the shape parameter $\gamma$ as fitted values for the shape parameter are close to this value in both 1979-1981 and 1989-1991 life tables, which cover periods close to those over which the Albertsen study (Albertsen et al, 1998, 2005) was conducted.

We calculated $\lambda_{\mathrm{O}}$ and $\lambda_{\mathrm{P}}$, conditional on $\gamma=1.8$, for each stratum using an iterative numerical algorithm written specifically for the task in Stata version 8.2 (Stata Corporation, College Station, TX, USA), the software used throughout this paper. The model relies in part on a reliable estimate of the shape parameter $\gamma$. The main end point, projected probability of survival at 15 years, however, appears largely insensitive to small variations in this parameter. Given a value for the parameter $(\gamma=1.8)$, values of $\lambda_{\mathrm{O}}$ and $\lambda_{\mathrm{P}}$ fitted to the data are detailed in Table 2.

\section{Step two: adapting the model to allow for PSA screening}

We adapted the model to allow for the lead time introduced by PSA screening and the probability of overdetection. There are no published data providing lead times and overdetection rates by Gleason score and by age at diagnosis. The lead-time values and overdetection proportions used for the model were taken as average values simulated by a replica of the Markov lead-time model fitted by Draisma et al (2003) for 'relevant' cases (detections that were not overdetections), stratified by age (within 5 years) and biopsy Gleason score $(<7,7,>7)$, assuming screening with $100 \%$ attendance every 2 years. The replica model was tested to assess how close its predictions of lead times and overdetection for various screening programmes were to those quoted from the original model (Draisma et al, 2003).

We averaged the fitted 15-year survival results from Albertsen et al (1998) in the Gleason score 2-4, 5 and 6 groups, weighted according to their sample size over those categories. By doing this, Gleason score categories in our model corresponded with those in Draisma et al (2003).

The survival outcomes for the overdetected proportion of cases were calculated assuming $h_{\mathrm{P}}(t)$ was equal to zero; for the 'relevant' cases, we calculated survival outcomes by setting $h_{\mathrm{P}}(t)$ to be zero for time $t$ less than the lead time, and equal to $\lambda_{\mathrm{P}}$ otherwise. For both groups, $h_{\mathrm{O}}(t)$ was left unchanged in this step by using the parameter values fitted in step one. Overall survival outcomes for screen-detected cases were calculated as the average of the two sets of survival outcomes figures, weighted according to the probability of being in each group.

\section{Step three: updating the model to a contemporary population}

Evidence of considerable reductions in mortality rates over the last 20 years can be found in US life tables (US Department of Health National Center for Health Statistics and Human Services). In fact, mortality at each age in every G7 country (Canada, France, Germany, Italy, Japan, UK, US) has declined exponentially in the last five decades at a roughly constant rate (Tuljapurkar et al, 2000). We accounted for mortality improvements in our modelling by multiplying fitted values of $\lambda_{\mathrm{O}}$ and $\gamma$, the two parameters in the other-cause mortality function, by values informed through the single-risk Weibull modelling of simulated life table survival data.

Table I Scale $(\times 100)$ and shape parameters fitted by the Weibull model of simulated survival from a period analysis of US life tables

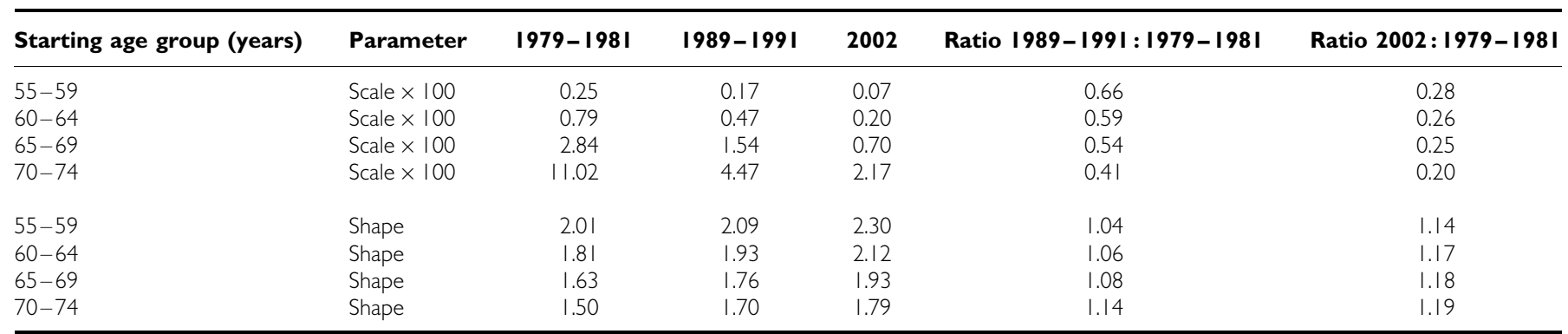

Table 2 Fitted values to the basic model from Albertsen's data of parameters $100 \times \lambda_{\circ}$ ('other') and $100 \times \lambda_{p}$ ('prostate') given $\gamma=1.8$

Age at diagnosis (years)

\begin{tabular}{|c|c|c|c|c|c|c|c|c|}
\hline \multirow[b]{2}{*}{ Gleason score } & \multicolumn{2}{|c|}{$55-59$} & \multicolumn{2}{|c|}{$60-64$} & \multicolumn{2}{|c|}{$65-69$} & \multicolumn{2}{|c|}{$70-74$} \\
\hline & Other & Prostate & Other & Prostate & Other & Prostate & Other & Prostate \\
\hline 7 & 0.32 & 9.84 & 0.52 & 8.62 & 0.82 & 7.58 & 1.31 & 6.37 \\
\hline$>7$ & 0.45 & 19.53 & 0.68 & 17.50 & 0.99 & 14.75 & 1.58 & 12.39 \\
\hline
\end{tabular}

$\lambda_{\mathrm{O}}=$ scale parameter; $\gamma=$ shape parameter. 
In the central projections, the multipliers used were 0.25 and 1.15 , respectively, chosen through consideration of the ratios shown in the final two columns of Table 1 .

\section{Step four: using the model to assess treatment benefit}

In order to assess the benefit of curative as opposed to conservative treatment, we required a model for mortality following curative treatment. The Scandinavian Prostatic Cancer Group Study assume in their analysis disease-specific hazards are proportional in relation to treatment (Bill-Axelson et al, 2005), an assumption we continued to adopt. We used their central estimate of the hazard ratio, 0.56, as the value for our parameter $H R$. We assumed a hazard function due to disease-specific mortality such that the disease-specific hazard ratio was equal to the parameter $H R$ for all age group and Gleason score strata at all times. The form of the hazard function $h_{\mathrm{Q}}(t)$ and its derivation are described in the Appendix A.

\section{Sensitivity analysis}

To estimate the sensitivity of our results to the choice of baseline treatment/data set, the procedure described above was repeated for summary data from a Mayo Clinic study by Sweat et al (2002) of the long-term survival with prostate cancer of 751 men after radical prostatectomy, the only research found in the literature providing the necessary cohort survival data in curatively treated men. The same value of $\gamma$ was used as before. New values of $\lambda_{\mathrm{O}}$ and $\lambda_{\mathrm{P}}$ were calculated based on reported fitted survival probabilities by age group and Gleason score strata at 20 years using Cox proportional hazards regression. As the base data relates to a cohort given curative rather than conservative treatment, $h_{\mathrm{P}}(t)$ was the disease-specific hazard function under curative treatment and $h_{\mathrm{Q}}(t)$ the disease-specific hazard function under conservative treatment with $H R$ now equal to $1.79(=1 / 0.56)$.

To assess the sensitivity of results to key parameters, we have also projected survival under 'low' and 'high' treatment benefit scenarios. Relative to the central scenario, the 'low treatment benefit' scenario assumes a higher overall survival hazard ratio for curative treatment (0.7), a lower improvement over time in other cause mortality ( $\lambda_{\mathrm{o}}$ multiplier of 0.7 and $\gamma$ multiplier of 1.3 ), longer lead times and greater overdetection rates; the high treatment benefit' scenario assumes a lower hazard ratio $(0.45)$, higher othercause mortality improvement $\left(\lambda_{\mathrm{o}}\right.$ multiplier of 0.1 and $\gamma$ multiplier of 1.0), shorter lead times and lower overdetection rates. The sensitivity ranges for the hazard ratio, lead time and overdetection rate were set as one standard deviation either side of the central estimate. In doing this, we assumed lead times, the logarithm of the hazard ratio and the logit of the overdetection rates were distributed normally.

\section{RESULTS}

\section{Constructing the model}

Average lead times and overdetection rates by age at diagnosis and by Gleason score from simulations of 1 million men with prostate cancer by the replica of Draisma's model (Draisma et al, 2003) for biennial screening are shown in Table 4.

The discrepancies between average lead times presented from Draisma's original model (Draisma et al, 2003) and our replica were found to be small: in the simulations, there was no more difference in the projected mean lead times for all cases and relevant cases only (cases of prostate cancer that would have been clinically detected) than 0.3 years for single screens, 0.2 years for schedules of annual screening and 0.3 years for schedules of quadrennial screening. The proportions of detections that were not 'relevant' agreed within $4 \%$ for all screening programmes.
For biennial PSA screening, the predicted average lead times for relevant cases were 9.9-14.1 years for cases with Gleason scores $<7,8.0-9.3$ years for those with Gleason score 7 and 5.0 -6.0 years for Gleason scores $>7$.

\section{Projection results}

Figure 2 shows projected survival over 15 years for contemporary patients with PSA screen-diagnosed prostate cancer managed conservatively, using the central model assumptions (based on data from Albertsen et al (1998), with 100\% attendance at biennial screening and multipliers for $\lambda_{\mathrm{O}}$ and $\gamma$ of 0.25 and 1.15 , respectively). Mortality is divided by projected cause (prostate cancer $v s$ other). Table 3 provides survival projections at 15 years sections (i)-(iii) of the table relate to the natural history of prostate cancer in conservatively treated men and section (iv) relates to survival in curatively treated men. The projected treatment effect, in terms of the absolute difference in 15-year survival, is shown in section (v).

Under the central assumptions based on conservative management, the probability of 15 -year mortality from prostate cancer appears strongly dependent on grade, particularly so for men who are younger at diagnosis. For example, for men aged 55-59 years at diagnosis, the projected 15 -year prostate cancer mortality is $0 \%$, $31 \%$ and $72 \%$ for men with Gleason scores $<7,7$ and $>7$, respectively. For men aged 70-74 years at diagnosis, the 15-year prostate cancer mortality is projected to be $2 \%, 9 \%$ and $28 \%$ for men with Gleason scores $<7,7$ and $>7$, respectively.

The projected overall survival benefit from curative treatment appears similarly dependent on Gleason score and age at diagnosis. For example, for men aged 55-59 years at diagnosis, the absolute 15 -year survival benefit from curative treatment is $0 \%, 12 \%$ and $26 \%$ for men with Gleason scores $<7,7$ and $>7$, respectively. For men aged 70-74 years at diagnosis, the 15-year overall survival benefit is projected to be $1 \%, 3 \%$ and $6 \%$ for men with Gleason scores $<7,7$ and $>7$, respectively.

\section{Sensitivity analyses}

Central projections of long-term survival probabilities together with the range of outcomes projected from the high and low scenarios using baseline data from either Albertsen et al (1998) or Sweat et al (2002) are shown in Table 4 with the ranges of lead times and overdetection rates assumed. While survival probabilities based on data from Sweat are generally higher than those based on data from Albertsen, likely due to differences in the casemix of the two studies, the point estimates are reasonably close and there is considerable overlap in the range of projected survival probabilities and absolute benefits, suggesting little sensitivity to baseline data set. Ranges of survival projections across scenarios are wide, but ranges of estimates of the absolute benefit due to treatment are small, suggesting little sensitivity of absolute benefit estimates to key parameter values. For example, the 15-year absolute benefit of curative treatment to a man diagnosed at age 60-64 years with a Gleason 7 cancer is 9\% (range across three scenarios: $0-32 \%$ ) based on Albertsen's data (Albertsen et al, 1998 ) and 4\% (range: $0-18 \%$ ) based on Sweat's data (Sweat et al, 2002).

\section{DISCUSSION}

This is the first study, to our knowledge, to model both the natural history of screen-detected prostate cancer and the impact of radical treatment on overall survival. While the outputs of the model must be interpreted with caution, they may have important implications for targeting treatment to those patients who stand to benefit most. Specifically, the absolute survival benefit of radical 
Age at diagnosis (years)
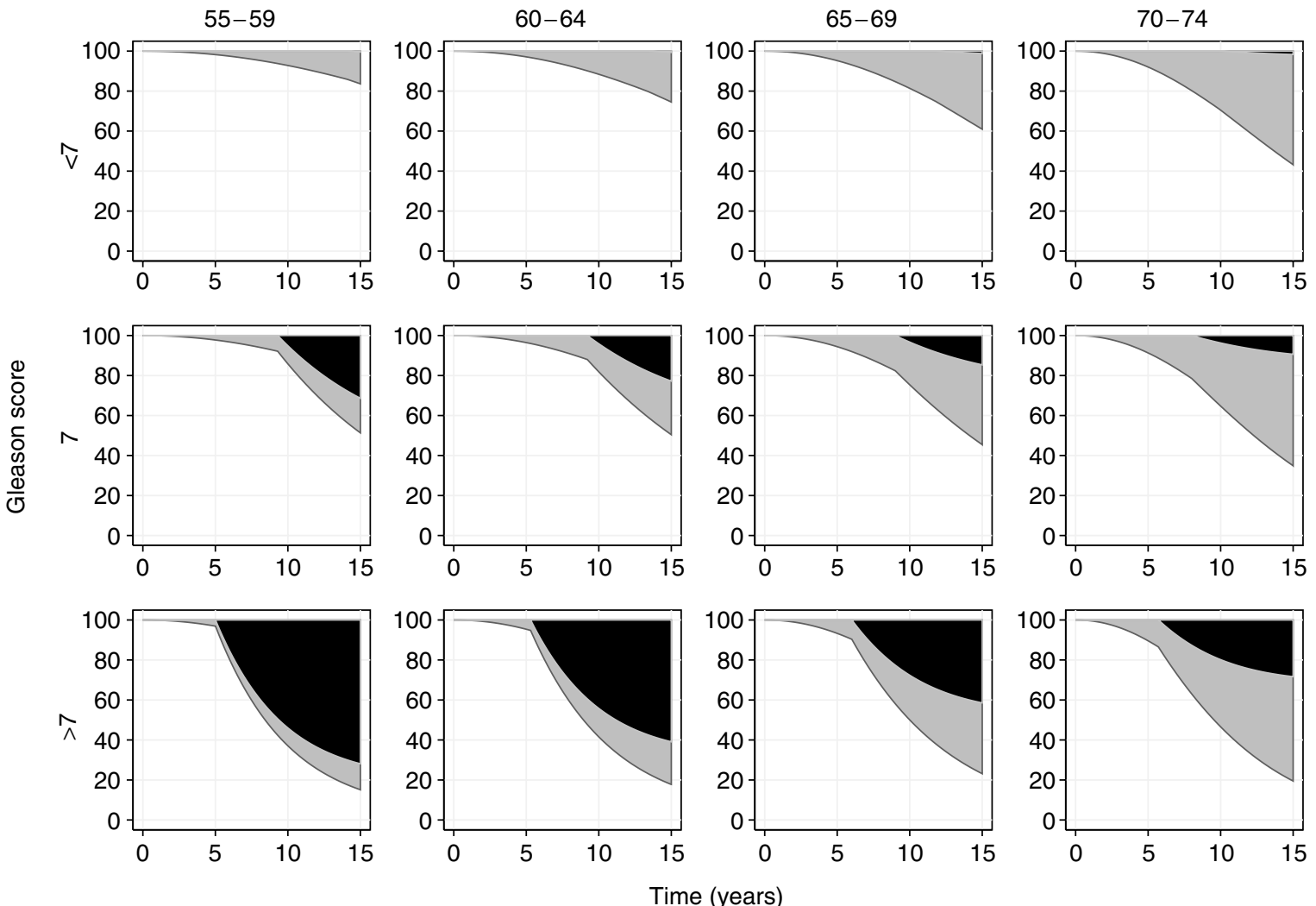

Figure 2 Projections of survival over 15 years under conservative treatment, using the central model assumptions. Black areas represent deaths due to prostate cancer, grey areas represent deaths due to other causes.

Table 3 Central projections of I5-year outcome probabilities and absolute benefit of treatment (to nearest whole percentage point). ('PC death': death attributed to prostate cancer)

\begin{tabular}{l} 
Age at diagnosis (years) \\
\hline $55-59$ \\
\hline
\end{tabular}

Gleason score Alive Other death PC death Alive Other death PC death Alive Other death PC death Alive Other death PC death

(i) Original data - conservative treatment

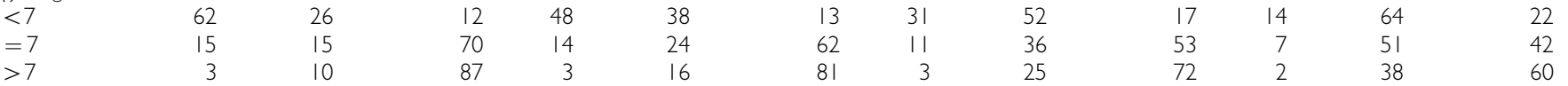

(ii) Conservative treatment, adjusting for screening (step two)

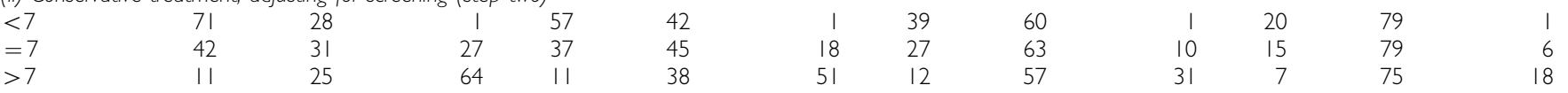

(iii) Conservative treatment, allowing for screening and contemporary population (step three)

$\begin{array}{rrrrrrrrrrrrr}<7 & 84 & 16 & 0 & 74 & 25 & 1 & 61 & 38 & 1 & 43 & 55 & 2 \\ =7 & 52 & 17 & 31 & 50 & 27 & 23 & 45 & 40 & 15 & 35 & 56 & 9 \\ >7 & 15 & 13 & 72 & 18 & 21 & 61 & 23 & 35 & 42 & 20 & 52 & 28\end{array}$

(iv) Curative treatment, allowing for screening and contemporary population (step four)

\begin{tabular}{|c|c|c|c|c|c|c|c|c|c|c|c|c|}
\hline$<7$ & 84 & 16 & 0 & 75 & 25 & 0 & 61 & 38 & 1 & 44 & 55 & I \\
\hline$=7$ & 64 & 18 & 18 & 59 & 28 & 13 & 51 & 41 & 8 & 38 & 57 & 5 \\
\hline$=7$ & 12 & 1 & -13 & 9 & I & -10 & 6 & I & -7 & 3 & 1 & -4 \\
\hline$>7$ & 26 & 6 & -32 & 20 & 7 & -27 & 12 & 7 & -19 & 6 & 6 & -12 \\
\hline
\end{tabular}


Table 4 Projections of I5-year survival probabilities based on data from Albertsen et al (1998) and Sweat et al (2002): central estimates and ranges of values obtained from low and high projections of life expectancy

\begin{tabular}{|c|c|c|c|c|c|c|c|c|c|}
\hline \multirow[b]{2}{*}{$\begin{array}{l}\text { Age group } \\
\text { (years) }\end{array}$} & \multirow[b]{2}{*}{$\begin{array}{l}\text { Gleason } \\
\text { score }\end{array}$} & \multicolumn{2}{|c|}{$\begin{array}{l}\text { Lead time } \\
\text { (years) }\end{array}$} & \multicolumn{2}{|c|}{$\begin{array}{l}\text { Overdetection } \\
\text { rate }(\%)\end{array}$} & \multicolumn{2}{|c|}{$\begin{array}{c}\text { Albertsen data: survival } \\
\text { benefit }(\%)\end{array}$} & \multicolumn{2}{|c|}{$\begin{array}{l}\text { Sweat data: survival } \\
\text { benefit (\%) }\end{array}$} \\
\hline & & Central & $\begin{array}{l}\text { Sensitivity } \\
\text { range }\end{array}$ & Central & $\begin{array}{l}\text { Sensitivity } \\
\text { range }\end{array}$ & Central & $\begin{array}{l}\text { Sensitivity } \\
\text { range }\end{array}$ & Central & $\begin{array}{c}\text { Sensitivity } \\
\text { range }\end{array}$ \\
\hline $55-59$ & $\begin{array}{l}<7 \\
=7 \\
>7\end{array}$ & $\begin{array}{r}14.1 \\
9.3 \\
5.0\end{array}$ & $\begin{array}{l}5.2-22.1 \\
2.8-15.1 \\
1.1-9.6\end{array}$ & $\begin{array}{r}37.1 \\
16.1 \\
7.3\end{array}$ & $\begin{array}{l}6.9-82.4 \\
1.2-74.5 \\
0.2-78.6\end{array}$ & $\begin{array}{r}0 \\
12 \\
26\end{array}$ & $\begin{array}{l}0-5 \\
0-36 \\
I-48\end{array}$ & $\begin{array}{r}0 \\
5 \\
12\end{array}$ & $\begin{array}{l}0-6 \\
0-17 \\
0-23\end{array}$ \\
\hline $65-69$ & $\begin{array}{l}<7 \\
=7 \\
>7\end{array}$ & $\begin{array}{r}11.8 \\
9.0 \\
6.0\end{array}$ & $\begin{array}{l}4.4-20.1 \\
2.7-15.2 \\
1.4-10.1\end{array}$ & $\begin{array}{l}66.9 \\
43.5 \\
25.8\end{array}$ & $\begin{array}{r}19.4-94.4 \\
9.3-85.3 \\
3.4-77.4\end{array}$ & $\begin{array}{r}0 \\
6 \\
12\end{array}$ & $\begin{array}{l}0-7 \\
0-27 \\
0-40\end{array}$ & $\begin{array}{l}0 \\
3 \\
6\end{array}$ & $\begin{array}{l}0-4 \\
0-15 \\
0-22\end{array}$ \\
\hline
\end{tabular}

treatment is predicted to be greater in men with high-grade disease, whereas current clinical practice preferentially targets radical treatment to patients with low-grade prostate cancer.

The central projections of the model based on Albertsen's data (Albertsen et al, 1998) are that the 15-year prostate-cancer-specific mortality for men aged 55-74 years diagnosed with screendetected prostate cancer with a Gleason score of $<7$, and managed conservatively, will be $1 \%$, and the absolute benefit in 15-year overall survival from curative treatment of such cases is predicted to be less than $1 \%$. The decision whether or not to have radical treatment is a value judgement, comparing the known morbidity of treatment with the potential survival benefit. Faced with a $30-60 \%$ risk of treatment-related impotence, and a $0-1 \%$ 15-year survival benefit, the majority of patients would decline radical treatment (Singer et al, 1991). In fact, data from the Cancer of the Prostate Strategic Urologic Research Endeavor (CaPSURE), an observational database of prostate cancer patients from 35 US centres, on 2078 cases diagnosed between 1989 and 2001 with low-risk prostate cancer (serum PSA $</=10 \mathrm{ng} \mathrm{ml}^{-1}$, Gleason sum $<I=6$ and clinical $T$ stage $<I=\mathrm{T} 2 \mathrm{a}$ ), show that just $7.9 \%$ of such men chose an observation policy, rather than treatment (Cooperberg et al, 2004). One explanation for this pattern of care is that patients and their clinicians have unrealistically high expectations of the survival benefit from treatment of low-risk disease, and this possibility deserves further study.

The model predicts that the absolute survival benefit of radical treatment for screen-detected prostate cancer is greater in highergrade disease. This finding is consistent with observations from retrospective studies. A population-based study from the SEER database, based on data for 59876 cancer-registry patients aged $50-79$ years who had clinically localised prostate cancer diagnosed between 1982 and 1992, analysed disease-specific survival by grade and by type of treatment (Lu-Yao and Yao, 1997) and found the absolute difference in 10-year disease-specific survival between radical prostatectomy and watchful waiting was 1,10 and $22 \%$ for grade 1,2 and 3 disease, respectively. Similarly, a multicentre comparison based on over 2000 patients, diagnosed between 1971 and 1984, observed a 10-year disease-specific survival difference between radical prostatectomy and expectant management of $4 \%$ for cases with Gleason score $2-4,8 \%$ for scores $5-6,17 \%$ for scores of 7 and 19\% for scores 8-10 (Barry et al, 2001). Patients who select watchful waiting are very different from those who select radical prostatectomy, and so retrospective comparisons between them should be interpreted with great caution. However, it is plausible that the differences between these two groups of patients are independent of tumour grade. So, taken together with the results of these retrospective studies, our model supports the hypothesis that the absolute survival benefit of radical treatment is greater for high-grade disease. These observations contrast with current patterns of care for men with localised prostate cancer. Of patients on the CaPSURE database with localised disease diagnosed from 1999 to 2001, primary treatment was with radical intent in 77.5, 75.8 and $47 \%$ of low-, intermediate- and high-risk cases, respectively (Cooperberg et al, 2003).

The majority of prostate cancers detected by PSA screening are low grade. Data on the grade-mix of screen-detected cancers from second round and subsequent screens shows $76-85 \%$ of cases with Gleason scores $<7,13-20 \%$ with a Gleason score of 7 and $1-4 \%$ with Gleason scores $>7$ (Hoedemaeker et al, 2001; Hugosson et al, 2004; Makinen et al, 2004). Based on these figures, and given the median age of detection of 67 years, our model predicts that the absolute 15-year overall survival benefit for radical treatment, compared with conservative management, of all screen-detected localised prostate cancers will be approximately $1-2 \%$. It is noteworthy that the ongoing clinical trials addressing this issue (PIVOT and ProtecT) are not adequately powered to detect a survival benefit of this magnitude.

Nicholson and Harland (2002) have previously modelled the natural history of screen-detected prostate cancer, based on temporal trends in prostate cancer incidence and mortality at a population level. They did not include consideration of the effect of age and grade on lead times, generational improvements in allcause mortality or the impact of treatment on prostate cancer outcomes. Notwithstanding these methodological differences, their projections of 15-year disease-specific mortality for screendetected cancers diagnosed between ages 55 and 74 years, and managed conservatively, ranged from 7.4 to $11.6 \%$, which are comparable to the current study.

The accuracy of our model is dependent on the validity of the underlying assumptions. To model the natural history of PSA screen-detected cancers, we have used published data to derive estimates, by patient age and Gleason score, for survival outcomes from expectant management in the pre-PSA era, and for lead times and overdetection rates associated with PSA screening. The 
Albertsen series $(1,8)$ used to describe the outcome of conservative management of localised disease from the pre-PSA era is highly regarded on account of its size, the maturity of the outcome data and the use of centralised pathology review with the Gleason scoring system. However, $70 \%$ of patients in that series did not have a staging bone scan, and there is evidence of systematic upgrading in the interpretation of the Gleason grading system over time (Smith et al, 2002; Kondylis et al, 2003). For both these reasons, the data from Albertsen are likely an overestimate, rather than an underestimate, of 'true' prostate cancer mortality from the pre-PSA era. In any event, using the Mayo Clinic radical prostatectomy data (Sweat et al, 2002), to describe the outcome of prostate cancer from the pre-PSA era, does not materially affect the outputs of the model. The model for estimating mean lead times and overdetection rates was derived from MIcrosimulation SCreening ANalysis (MISCAN) simulations of results validated against data from the Rotterdam section of the European Randomised Study of Screening for Prostate Cancer (ERSPC), which enrolled 21166 men in the unscreened arm and 21210 men in the screened arm and in which 1498 prostate cancers were diagnosed. The Rotterdam screening policy evolved during the study, but predominantly consisted of a sextant prostate biopsy for men with a PSA $>3 \mathrm{ng} \mathrm{ml}^{-1}$. MISCAN models are based on Markov processes of states and transitions and are designed to evaluate cancer-screening programmes.

To model the absolute survival benefit of curative treatment in the context of PSA screen detection, we used data from the only randomised trial available, the Scandinavian Prostatic Cancer Group Study. The hazard ratio for the effect of radical treatment on prostate cancer mortality was taken to be 0.56 (95\% CI: $0.36-0.88$ ), and this hazard ratio was assumed to be independent of tumour grade and patient age. Subgroup analyses of the Scandinavian trial are consistent with these assumptions (Bill-Axelson et al, 2005). However, the trial consisted predominantly of patients with lowgrade disease, and included just 159 cases (23\%) with Gleason score 7, and 35 cases (5\%) with Gleason score $>7$. A further assumption is that the hazard ratio for the benefit of curative treatment on prostate cancer mortality, derived from data on patients with cancers typical of the pre-PSA era, is applicable to patients with screen-detected cancers. In the absence of any data on this issue, this is a significant limitation.

\section{CONCLUSIONS}

This modelling exercise, based on published data, describes the natural history of screen-detected prostate cancer and the impact of radical treatment on overall survival. The results of the model should be interpreted with caution, since the original data upon which it is based cannot necessarily be assumed to be generalisable more widely. With that proviso, the 15-year mortality from lowgrade, screen-detected prostate cancer in men aged 55-74 years at diagnosis, who elect conservative management, is projected in our central model to be $1 \%$, and the absolute 15 -year survival benefit of curative treatment, less than $1 \%$. The absolute survival benefit for radical treatment is predicted to be greater in men with high-grade disease. When the results of PIVOT and the ProtecT trial are mature, subgroup analysis by grade will be important. Until then, the predictions of our model, together with the available retrospective data, suggest that the case for radical treatment, rather than conservative management, of localised prostate cancer is strongest in men with high-grade disease.

\section{ACKNOWLEDGEMENTS}

This work was undertaken in The Royal Marsden NHS Foundation Trust who received a proportion of its funding from the NHS Executive; and in the Cancer Screening Evaluation Unit which receives funding from the Department of Health; the views expressed in this publication are those of the authors and not necessarily those of the NHS Executive or the Department of Health. This work was supported by the Institute of Cancer Research, the Cancer Research UK Section of Radiotherapy [CUK] grant number C46/A2131 and NCRI South of England Prostate Cancer Collaborative.

\section{REFERENCES}

Albertsen P, Gleason D, Barry M (1998) Competing risk analysis of men aged 55 to 74 years at diagnosis managed conservatively for clinically localized prostate cancer. JAMA 280: $975-980$

Albertsen PC, Hanley JA, Fine J (2005) 20-year outcomes following conservative management of clinically localized prostate cancer. JAMA 293: $2095-2101$

Anderson P, Abildstrom S, Rosthoj S (2002) Competing risks as a multistate model. Stat Methods Med Res 11: 203-215

Barry MJ, Albertsen PC, Bagshaw MA, Blute ML, Cox R, Middleton RG, Gleason DF, Zincke H, Bergstralh EJ, Jacobsen SJ (2001) Outcomes for men with clinically nonmetastatic prostate carcinoma managed with radical prostactectomy, external beam radiotherapy, or expectant management: a retrospective analysis. Cancer 91: 2302-2314

Bill-Axelson A, Holmberg L, Ruutu M, Haggman M, Andersson SO, Bratell S, Spangberg A, Busch C, Nordling S, Garmo H, Palmgren J, Adami HO, Norlen BJ, Johansson JE (2005) Radical prostatectomy versus watchful waiting in early prostate cancer. $N$ Engl J Med 352: $1977-1984$

Cooperberg MR, Grossfeld GD, Lubeck DP, Carroll PR (2003) National practice patterns and time trends in androgen ablation for localized prostate cancer. J Natl Cancer Inst 95: 981 -989

Cooperberg MR, Lubeck DP, Meng MV, Mehta SS, Carroll PR (2004) The changing face of low-risk prostate cancer: trends in clinical presentation and primary management. J Clin Oncol 22: 2141-2149

Donovan J, Hamdy F, Neal D, Peters T, Oliver S, Brindle L, Jewell D, Powell P, Gillatt D, Dedman D, Mills N, Smith M, Noble S, Lane A (2003) Prostate Testing for Cancer and Treatment (ProtecT) feasibility study. Health Technol Assess 7: 1-88
Draisma G, Boer R, Otto S, van der Cruijsen I, Damhuis R, Schroder F, Koning H (2003) Lead times and overdetection due to prostate-specific antigen screening: estimates from the European Randomized Study of Screening for Prostate Cancer. J Natl Cancer Inst 95: 868-878

Hoedemaeker RF, van der Kwast TH, Boer R, de Koning HJ, Roobol M, Vis AN, Schroder FH (2001) Pathologic features of prostate cancer found at population-based screening with a four-year interval. J Natl Cancer Inst 93: $1153-1158$

Hugosson J, Aus G, Lilja H, Lodding P, Pihl CG (2004) Results of a randomized, population-based study of biennial screening using serum prostate-specific antigen measurement to detect prostate carcinoma. Cancer 100: $1397-1405$

Iversen P, Madsen PO, Corle DK (1995) Radical prostatectomy versus expectant treatment for early carcinoma of the prostate. Twenty-three year follow-up of a prospective randomized study. Scand J Urol Nephrol Suppl 172: $65-72$

Kondylis FI, Moriarty RP, Bostwick D, Schellhammer PF (2003) Prostate cancer grade assignment: the effect of chronological, interpretive and translation bias. J Urol 170: 1189-1193

Lu-Yao G, Yao S (1997) Population-based study of long-term survival in patients with clinically localised prostate cancer (see comments). Lancet 349: $906-910$

Makinen T, Tammela TL, Stenman UH, Maattanen L, Aro J, Juusela H, Martikainen P, Hakama M, Auvinen A (2004) Second round results of the Finnish population-based prostate cancer screening trial. Clin Cancer Res 10: $2231-2236$

Nicholson PW, Harland SJ (2002) Survival prospects after screen-detection of prostate cancer. BJU Int 90: 686-693 
Singer PA, Tasch ES, Stocking C, Rubin S, Siegler M, Weichselbaum R (1991) Sex or survival: trade-offs between quality and quantity of life. J Clin Oncol 9: $328-334$

Smith EB, Frierson Jr HF, Mills SE, Boyd JC, Theodorescu D (2002) Gleason scores of prostate biopsy and radical prostatectomy specimens over the past 10 years: is there evidence for systematic upgrading? Cancer 94: $2282-2287$

Sweat SD, Bergstralh EJ, Slezak J, Blute ML, Zincke H (2002) Competing risk analysis after radical prostatectomy for clinically nonmetastatic prostate adenocarcinoma according to clinical Gleason score and patient age. J Urol 168: 525-529

Tuljapurkar S, Li N, Boe C (2000) A universal pattern of mortality decline in the G7 countries. Nature 405: 789-792

\section{Appendix A}

Marginal survival from death due to prostate cancer to time $t$ since diagnosis under conservative treatment is $S_{\mathrm{P}}(t)=\exp \left(-\lambda_{\mathrm{P}} t\right)$. Given a hazard ratio of $H R$, survival from death due to prostate
US Department of Health National Center for Health Statistics and Human Services (2004) National Vital Statistics Reports: United States life tables 2002 http://www.cdc.gov/nchs/data/nvsr/nvsr53/nvsr53_06.pdf

US Department of Health National Center for Health Statistics and Human Services (1985) US Decennial life tables for 1979-1981. http:// www.cdc.gov/nchs/data/lifetables/life79_11.pdf

US Department of Health National Center for Health Statistics and Human Services (1997) US Decennial life tables for 1989-1991. http:// www.cdc.gov/nchs/data/lifetables/life89_1_1.pdf

Wilt TJ, Brawer MK (1995) The Prostate Cancer Intervention Versus Observation Trial (PIVOT): A randomized trial comparing radical prostatectomy versus expectant management for the treatment of clinically localised prostate cancer. Cancer 75: 1963-1968

cancer to time $t$ since diagnosis under curative treatment is $S_{\mathrm{Q}}(t)=1-H R \times\left[1-\exp \left(-\lambda_{P} t\right)\right]$. Noting that (Anderson et al, 2002) $\quad h_{\mathrm{Q}}(t)=-\mathrm{d} / \mathrm{d} t \log S_{\mathrm{Q}}(t)$, we obtain $h_{\mathrm{Q}}(t)=\left\{H R \lambda_{\mathrm{P}} \exp \right.$ $\left.\left(-\lambda_{\mathrm{P}} t\right)\right\} /\left\{1-H R \times\left[1-\exp \left(-\lambda_{P} t\right)\right]\right\}$ 